\title{
EDITORIAL
}

\section{¿Y QUÉ ES ESO DE LOS DETERMINANTES SOCIALES DE LA SALUD?}

\author{
Mauricio Hernando Torres-Tovar \\ Médico, Magister en Salud Pública, estudiante de Doctorado en Salud Pública \\ Profesor Asistente, Departamento de Salud Pública, Universidad Nacional de Colombia \\ Profesor Posgrados en Salud, Facultad de Ciencias Sociales, Universidad Colegio Mayor de Cundinamarca
}

Los desarrollos del campo de la epidemiología en su objeto de interpretar la dinámica de los procesos de enfermedad de grupos poblacionales llevó a comprender que no era posible explicar las causas de las enfermedades solo como fenómenos de orden biológico. El enfoque de los determinantes sociales de la salud ha venido a contribuir con la identificación de las causas de los perfiles epidemiológicos y de las que establecen la distribución desigual de la salud, la enfermedad y la muerte en la sociedad.

No hay un concepto univoco de determinantes sociales de la salud. El concepto de manera general se refiere a que la salud no tiene una causa exclusivamente biológica, sino que existen múltiples condiciones, fundamentalmente de carácter económico y social, que definen los procesos de salud-enfermedad de las personas y los grupos sociales ${ }^{1-5}$; razón por lo cual no basta solamente con intervenir en el plano de las condiciones biológicas desde los servicios de salud, sino que se requiere de intervenciones en los otros ordenes señalados para lograr mejores condiciones de salud para la población y para reducir las desigualdades en salud injustas y evitables ${ }^{6,7}$.

\section{Vertientes interpretativas de los determinantes de las inequidades sanitarias}

Hoy en día el centro del interés está más en el tema de los determinantes sociales de las inequidades de la salud. Existen diversos modelos que buscan comprender la relación entre determinantes y la producción de inequidades en salud ${ }^{8-10}$, entre los que sobresalen:
1. Niveles de influencia: desarrollado por Whitehead y Dahlgren ${ }^{11}$. Plantea que hay determinantes en diferentes niveles, un nivel más cercano a los determinantes individuales y otro más distal en donde se encuentra los macro-determinantes y que son estos los que definen los estados de salud de las personas.

2. Estratificación social: desarrollado por Diderichsent y colaboradores $^{12}$. Hacen hincapié en la estratificación social que da a las personas diferentes posiciones sociales, las cuales producen diferencias de salud. Cada individuo ocupa una cierta posición social, como resultado de diversos mecanismos sociales, tales como el sistema educativo y el mercado laboral. De acuerdo con la posición social ocupada por diferentes individuos se tienen exposiciones diferenciales a riesgos, vulnerabilidad diferencial a la aparición de la enfermedad y respuestas diferenciales para su enfrentamiento.

3. Influencias múltiples en el ciclo de vida: desarrollado principalmente por Marmot y Wilkinson ${ }^{3,4}$. El modelo ilustra como las circunstancias sociales y económicas de las personas afectan fuertemente su salud durante la vida, razón por la cual las desigualdades socio-económicas generan un diferencial de exposición a riesgos a lo largo del ciclo de la vida que generan un diferencial en las condiciones de salud de las poblaciones.

4. Niveles de determinantes: desarrollado por la Comisión de Determinaste Sociales de la Salud de la $\mathrm{OMS}^{1}$. Según este modelo existen dos tipos de determinantes sociales, los estructurales ligados al contexto macro socioeconómico y político que definen la posición social de los individuos y por lo tanto establece la inequidades en salud y los 
intermedios relacionados con las condiciones materiales de vida y trabajo definidas por las condiciones de las personas según su educaciónocupación-ingreso.

5. Determinación de la salud: desarrollado principalmente por Jaime Breihl ${ }^{2}$, como parte de la corriente de medicina social latinoamericana. Este modelo considera que existe una determinación de la salud en las esferas general, particular e individual, definidas por el modelo de desarrollo económico de la sociedad que impone un cierto tipo de procesos productivos y reproductivos, y que define la clase social de los individuos producto de las formas de distribución de la riqueza, estableciendo el modo de andar por la vida que define o a más factores protectores o a más factores deteriorantes para su salud, configurando los perfiles epidemiológicos de las poblaciones.

Estos modelos explicativos de los determinantes de las inequidades tienen en común un reconocimiento de la posición social como base explicativa de las desigualdades presentes en salud en las sociedades. Las diferencias radican de un lado en que unos se ciñen solo a evidenciar cuáles son los determinantes, convirtiéndolos de esta manera en una categoría de factores de riesgo, mientras que otros buscan explicar las relaciones y mediaciones entre los diferentes niveles y el origen de las desigualdades.

De otro lado, difieren en cómo afectar las inequidades, para la mayoría se trata de mejorar el acceso a la educación para mejorar la ocupación y así obtener más ingresos para cambiar la posición social, en tanto la otra vertiente al hablar de una determinación social considera que hay que afectar la matriz del modelo de desarrollo económico para cambiar las formas de distribución de la riqueza y las relaciones de poder dadas por la clase social, el género y la etnia, bases estructurales de la inequidad en salud ${ }^{2,5}$.

\section{La Salud Pública debe incorporar este enfoque}

Dada la situación de inequidades en salud presentes tanto en el mundo como en Colombia, debe ampliar seel desarrollo, apropiación y uso del enfoque de determinantes, con la clara intención de contribuir a la garantía del derecho a la salud de las poblaciones ${ }^{13}$.
La ventana de oportunidad abierta por la Comisión de Determinantes Sociales de la Salud de la $\mathrm{OMS}^{2}$ debe ser aprovechada para continuar trabajando con intensidad en los próximos años en este enfoque, buscando aportar en el reconocimiento e intervención de las causas que generan las desigualdades en salud.

La salud pública debe aprovechar este enfoque para trabajar en la generación de conocimiento que logren influir en la praxis de los salubristas públicos y en el diseño de políticas públicas en salud; lo que puede contribuir a fortalecer su campo disciplinar y a participar de manera protagónica en la acción intersectorial e interdisciplinaria que demanda la tarea de superar las condiciones de enfermedad injustas y evitables presentes en la sociedad.

\section{Referencias Bibliográficas}

1. Organización Mundial de la Salud. Comisión de Determinantes Sociales de la Salud. Subsanar las desigualdades en una generación. Alcanzar la equidad sanitaria actuando sobre los determinantes sociales de la salud. Buenos Aires: Ediciones Journal S.A.; 2009.

2. Breihl J. Epidemiologia Crítica: ciencia emancipadora e interculturalidad. Buenos Aires: Lugar editorial, 2003.

3. Marmot M. The status syndrome: how social standing affects our health and longevity. New York: Times Books; 2004.

4. Wilkinson R. Unhealthy societies: the afflictions of inequality. London: Routledge; 1996.

5. Eibenschuzt C, Tamez S, González R (compiladores). ¿Determinación social o determinantes sociales de la salud? Ciudad de México: Universidad Autónoma Metropolitana; 2011.

6. Berlinguer G. Determinantes de la enfermedad. Rev. Cubana Salud Pública. 2007; 37(1).

7. Navarro V. What we mean by social determinants of health. International Journal of Health Services. 2009; 39(3).

8. Buss P, Pellegrini A. Saúde e seus Determinantes Sociais. Rev. Saúde Coletiva. 2007; 17(1).

9. Álvarez LS. Los determinantes sociales de la salud: más allá de los factores de riesgo. Rev. Gerenc. Polit. Salud. 2009; 8 (17).

10. Moiso A. Determinantes de la salud. En: Barragán LH. Fundamentos de Salud Pública. La Plata: Editorial Universidad Nacional de La Plata; 2007. 
11. Whitehead M, Dahlgren G. Concepts and principles for tackling social inequities in health: Levelling up Part 1. Copenhagen: World Health Organization. Regional Office for Europe; 2006.

12. Diderichsen P, Evans T and Whitehead M. The social basis of disparities in health. En: Evans et al. Challenging inequities in health: from ethics to action. New York: Oxford UP; 2001. pp. 15-32.
13. Torres-Tovar M. Los determinantes sociales de la salud: herramienta para la acción política por el derecho a la salud. En: Eibenschuzt C, Tamez S, González R (compiladores). ¿Determinación social o determinantes sociales de la salud? Ciudad de México: Universidad Autónoma Metropolitana; 2011. pp. 207-224. 